\title{
Interlenticular opacification in piggyback AcrySof intraocular lenses: explantation technique and laboratory investigations
}

\author{
Haralabos Eleftheriadis, Julia Marcantonio, George Duncan, Christopher Liu
}

\begin{abstract}
Backgroundlaims-Interlenticular opacification (ILO) is a recognised complication of piggyback intraocular lenses (IOLs). The aetiology, histopathology, and treatment are not clearly defined, however.

Methods-Two pairs of AcrySof IOLs were explanted from a patient with bilateral ILO. The explantation technique and surgical challenges of IOL exchanges are described. The explanted IOL complexes and a sample of the anterior capsule were examined by phase, polarising, and immunofluorescence microscopy.

Results-A 50 year old man developed ILO bilaterally after piggyback AcrySof IOL implantation. A central contact zone was surrounded by a homogeneous paracentral opacity possibly consisting of extracellular matrix previously laid down by proliferating lens epithelial cells (LECs). These opacities were in turn surrounded by interlenticular Elschnig pearl-type opacities contiguous with the same material filling the periphery of the capsular bag. The IOL complexes were very adherent to the capsular bag and they had to be separated with the help of high viscosity viscoelastic before a single one piece PMMA IOL implantation via large limbal incisions. The sample of anterior capsule showed a ridge configuration from the piling of LECs in the site of apposition with the anterior capsule and cells showing different characteristics on either side of the ridge.
\end{abstract}

Conclusion-Cellular proliferation, deposition of ECM from proliferating LECs, and capsular changes induced by cell metaplasia may lead to ILO formation in piggyback AcrySof IOLs. Careful separation of the AcrySof IOL complex from the capsule, meticulous clean up of the proliferating material, and implantation of single or dual in the bag PMMA IOLs through a large incision with capsulorrhexis enlargement may help in the prevention of recurrence of interface opacification.

(Br f Ophthalmol 2001;85:830-836)

Gayton and Saunders first introduced polypseudophakia in 1993 in a microphthalmic patient with cataract to overcome the available power of a single intraocular lens (IOL). ${ }^{1}$ Since then many surgeons have adopted this practice. $^{2}{ }^{3}$ However, it appears that a visually significant complication named interlenticular opacification (ILO), ${ }^{4}$ otherwise known as interpseudophakos opacification (IPO), ${ }^{5}$ can develop in some cases. ILO is characterised by opacification in the interlenticular interface caused by Elschnig pearls or membrane formation, resulting in a loss of best corrected visual acuity (BCVA) and hyperopic shift. ${ }^{4-6}$ It usually occurs 1-2 years after primary "in the bag" implantation of piggyback IOLs. ILO is more common in patients with AcrySof IOLs than with silicone or PMMA IOLs implanted in the capsular bag ${ }^{4}$ and it has not to date been reported with primary or secondary piggyback IOLs in the ciliary sulcus. ${ }^{5}$

Three types of IPO have been described by Shugar ${ }^{7}$ according to the material seen between the IOLs: (a) IPO-E, where Elschnig pearl-type material grows in the interlenticular space from the periphery and is associated with hyperopic shift and reduced vision, necessitating IOL exchange, (b) IPO-A, which is similar to anterior capsular fibrosis and can be associated with central membrane formation, and (c) IPO-P, a proteinaceous acellular material, which may be very adherent to the central interface.

The development of opacities in the interlenticular space appears to be the result of proliferation and migration of residual lens epithelial cells (LECs) in the capsular bag. ${ }^{4}$ We describe here the surgical technique and visual and refractive results of exchange of piggyback AcrySof IOLs from both eyes of one patient affected by ILO. The explanted IOLs and a piece of anterior capsule were then investigated by light microscopy and other cell biological techniques.

\section{Clinical details}

A 50 year old man presented in October 1999 complaining of gradual deterioration of vision bilaterally over the preceding year and considerable annoying glare in bright light and at night. Initially his vision improved with hypermetropic spectacles, which needed to become progressively stronger. However this was not sufficient to alleviate the glare.

Two years earlier he was found to have bilateral cataracts and marked hypermetropic astigmatism. He underwent elsewhere a left phacoemulsification with implantation of piggyback acrylic IOLs (+15.5 and +16.50 dioptres (D) AcrySof MA30BA, Alcon), followed by a right phacoemulsification with piggyback acrylic
Accepted for publication 26 February 2001 
(+15.0 D and +16.50 D AcrySof MA30BA) IOLs 1 week later. Approximately 1 month later he underwent bilateral paired arcuate keratotomies in the $7 \mathrm{~mm}$ corneal zone in order to reduce $>3 \mathrm{D}$ of corneal astigmatism. $\mathrm{He}$ was very pleased with his vision and was able to see very well for distance without spectacles (refraction not available).

When he presented to us in October 1999, his visual acuity was $6 / 18$ unaided (6/5 with $+2.25+0.25 \times 160)$ in the right eye and $6 / 24$ unaided $(6 / 9-3$ with $+2.75+1.00 \times 129)$ in the left. Both corneas showed superior and inferior arcuate keratotomies. The anterior chambers were deep and quiet and the pupils were normal. The anterior capsules did not show any evidence of fibrosis and the capsulorrhexes were overlapping the optic of the anterior IOLs. Both IOLs were in the capsular bag with their haptics almost aligned in the horizontal meridian in the left eye (Fig 1a and b) and about 30 degrees apart in the right eye (Fig 1c). Elschnig pearl-type opacities were present in the peripheral interlenticular spaces in both eyes extending up to the mid-periphery. There
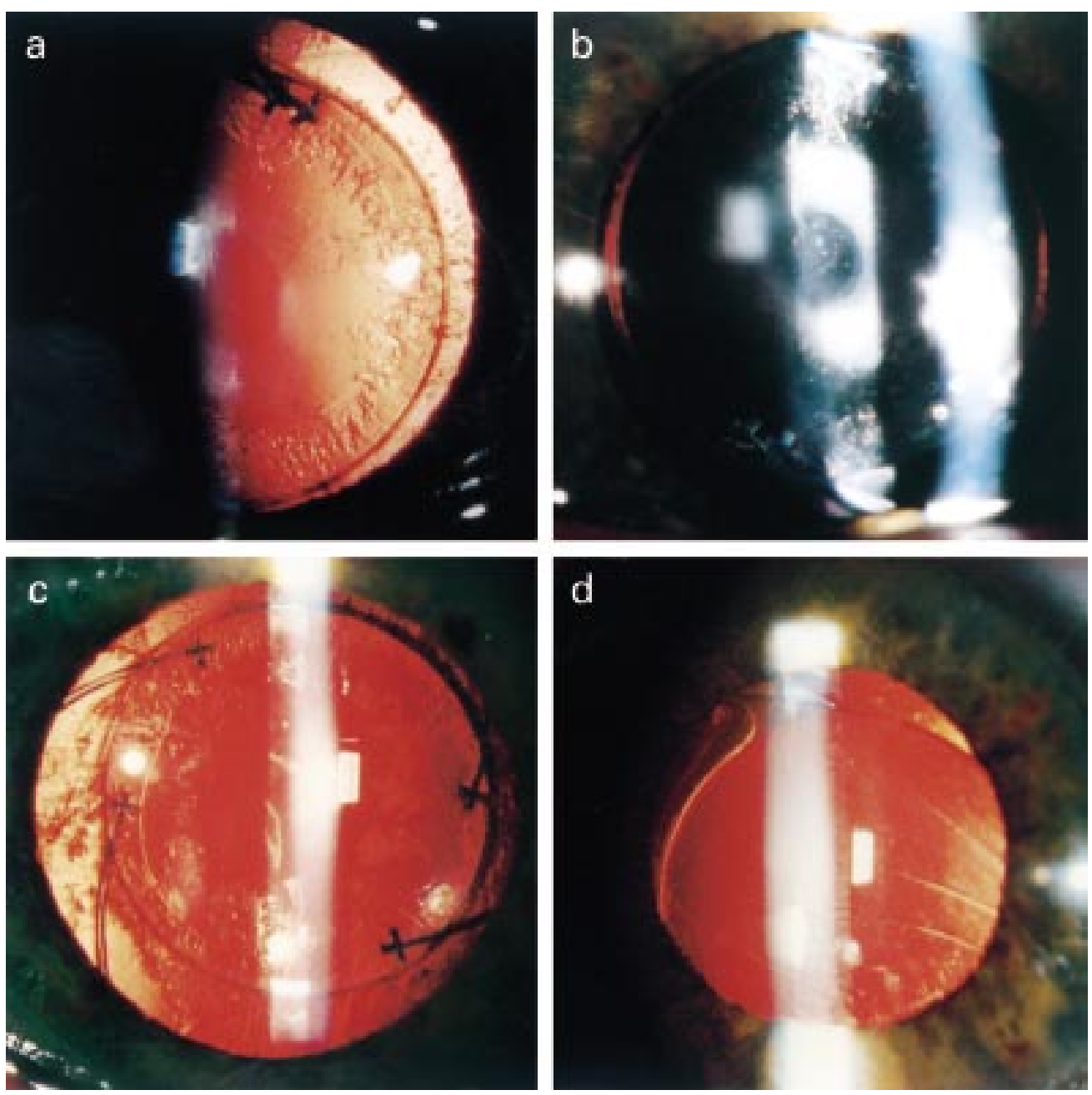

Figure 1 (a) and (b) Slit lamp photographs of preoperative appearance of left eye. Note clear central zone, opacified mid-zone, and peripheral strands of pearl-like material in the interlenticular space extending into the peripheral capsular bag space. The anterior capsulorrhexis can be seen against the red reflex (a). The two lens implants are well aligned. (c) Slit lamp photograph of preoperative appearance of right eye. There is less interlenticular opacification in this eye. The haptics are not aligned in this case but the optics are. (d) Slit lamp photograph of post-exchange of IOL appearance of left eye. The PMMA IOL is well centred within the capsular bag. The anterior capsulorrhexis edge is just visible. The posterior capsule has developed some folds. 


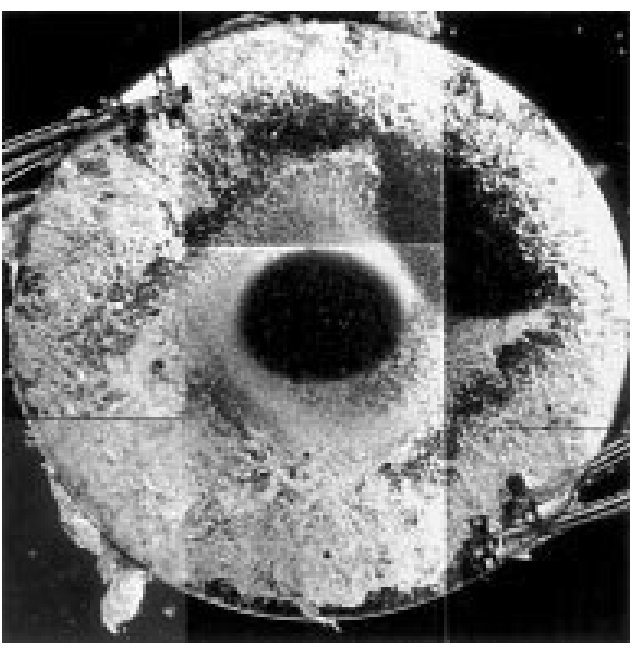

Figure 2 Photomontage of low magnification light micrographs to show overall structure of the fixed IOL pair before separation. Cellular and extracellular material between the optics appears white owing to light scatter. A central clear zone is surrounded by an area of homogeneous light scatter and this in turn is surrounded by a region of intense light scatter from Elschnig pearl-like material.

to a viscoelastic filled syringe was placed between the space opened between the capsule and the optic. The capsular bag was thus inflated with viscoelastic injected inferiorly followed by superior inflation with a 25 gauge Corydon cannula. The fused anterior and posterior capsules outside the optical zone were thus relatively easily separated by the injected viscoelastic. The posterior IOL remained adherent to the posterior capsule and required further injection of Healonid GV via a Rycroft cannula introduced directly behind the posterior IOL. The haptics were strongly adherent to or entrapped on the equator of the capsular bag and a Sinskey hook was used to dial them free. The two IOLs were fused together and their separation was not attempted. The piggyback complex, completely free from any capsular adhesions, was rotated out of the bag into the posterior chamber. A lens glide (Visitec, model Hessburg) was introduced behind the IOLs to facilitate their removal and to prevent tearing of the posterior capsule by the haptics. The IOL complex was retrieved from the eye, applying some pressure on the posterior lip of the section in order to keep the IOLs away from the corneal endothelium. The capsular bag equator contained material similar to soft lens matter found in cataract surgery and this was aspirated meticulously. The posterior capsule was free from opacities. After reinflation of the bag and anterior chamber with further viscoelastic, a single $+30 \mathrm{D}$ one piece PMMA IOL was inserted in the capsular bag and a small piece of anterior capsule was removed for histological studies. As the peripheral iris was prolapsing through the limbal wound, a peripheral iridectomy was performed. The wound was closed with interrupted 10-0 nylon sutures.

The explanted IOLs and anterior capsule fragment were placed in fixative ( $4 \%$ formaldehyde in phosphate buffered saline). After 1 hour, the fixative strength was reduced to $1 \%$ for transport of specimens to the laboratory.
In April 2000 the patient underwent a similar procedure on the right eye with explantation of the AcrySof IOLs and implantation of a one piece +30 D PMMA IOL in the capsular bag. At the last postoperative visit (June 2000), 10 weeks after the right IOL exchange and 8 months after the left, the BCVA was $6 / 5$ on the right eye with $-1.50+2.25 \times 125$ and $6 / 9$ with $-0.50+1.50 \times 45$ on the left (Fig 1d).

\section{Laboratory findings}

The IOLs and capsular fragment were observed by phase and polarising microscopy and photographed (Figs 2-5). The IOLs were separated, with difficulty, by inserting a fine blade between them. Some interlenticular material was left on each IOL, and cellular material was attached to the haptics. Indirect immunocytochemical staining was carried out for vimentin (an intermediate filament marker for LECs) and $\alpha$ smooth muscle actin ( $\alpha$-sma, a marker for epithelial-mesenchymal transition). Samples were also stained with phalloidin for F-actin and DAPI for cell nuclei.

When the fixed IOL assembly was examined by phase microscopy the regions of opacification appeared white due to light scatter (Figs 2 and $3 a$ ). The light scattering material was of two distinct types. A region with a regular cobblestone appearance surrounded the clear central area (Fig 3b). The regular hexagonal outlines (Fig 3b inset) gave the appearance of an epithelial sheet, but staining revealed no remaining cytoskeletal or nuclear elements. This material is likely to be the remains of extracellular matrix (ECM) secreted by a population of LECs that had migrated into the interlenticular space at an early stage of ILO development. The space between the outer edges of the IOL optics was filled with pearl-like and globular material (Fig 3c). This material stained for both $\mathrm{F}$-actin and $\alpha$-sma (Fig 3d) with the F-actin forming a submembranous layer round the globules. Moreover, DAPI staining revealed the presence of large numbers of cell nuclei (Fig 3e) scattered throughout the globular material. Higher magnification (Fig 3f) showed that many of the nuclei were degenerate and some micronuclei were present.

The capsular fragment had a full covering of cells except for the rhexis edge, where cells may have been disrupted by the surgical explantation. The most striking feature (Fig 4a) was the presence of a prominent light scattering ridge running parallel to the curved capsulorrhexis edge. Polarising microscopy revealed that this ridge showed strong birefringence (Fig 4b) indicating alignment of cells and/or macromolecules within its structure. Similar birefringent structures with aligned cells have been observed at PMMA/capsule interfaces in donor capsular bags with single implanted IOLs. ${ }^{89}$ Viable cells were present within the ridge at the time of fixation, and cell nuclei were elongated and aligned along the length of the ridge (Fig $4 d)$.

Cell morphology and $\alpha$-sma expression were different on each side of the ridge. Cells on the IOL flap between the ridge and the rhexis edge 

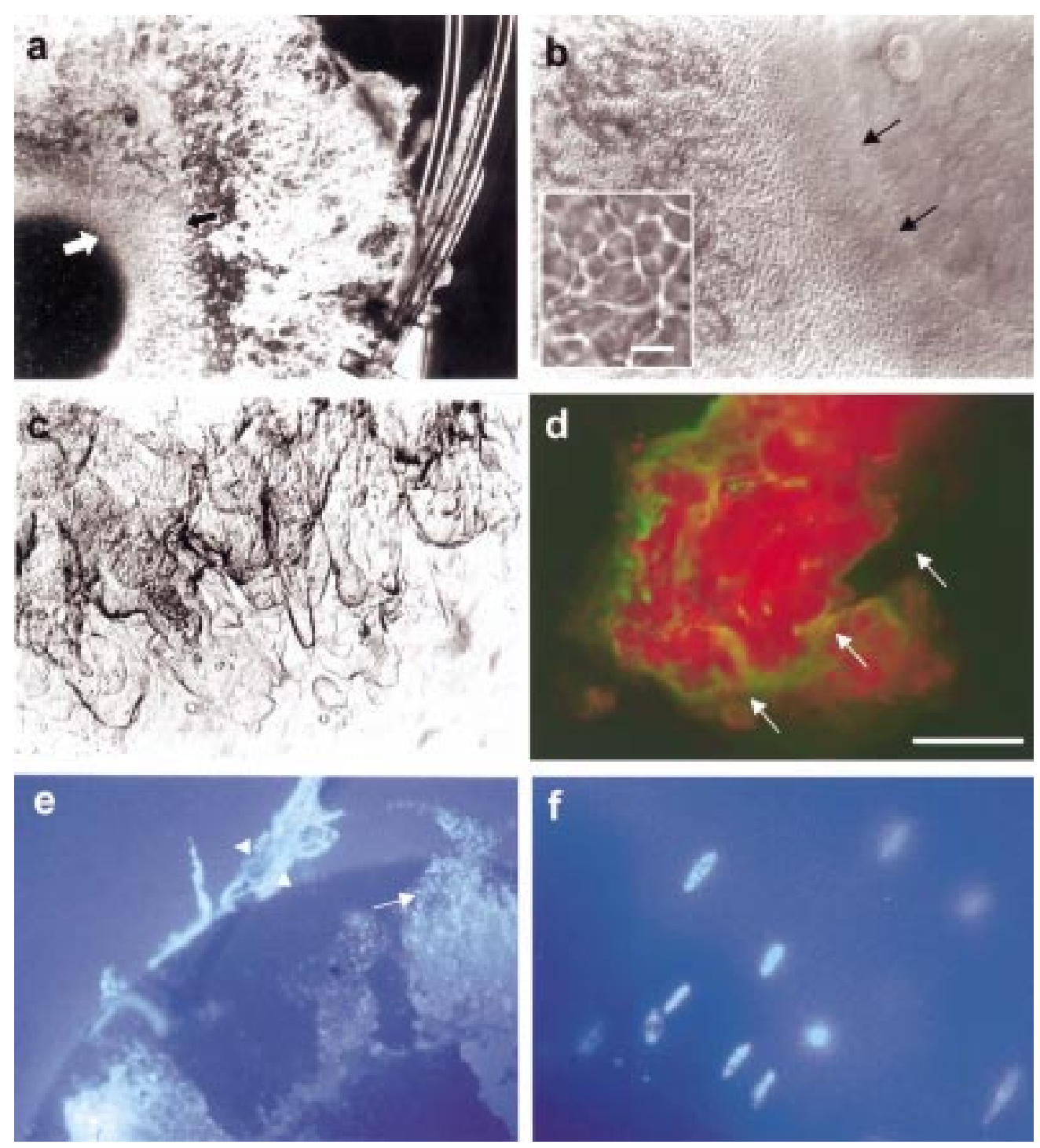

Figure 3 Images of the IOLs. (a) Low magnification overview of fixed IOLs before separation. Material can be seen adhering to both the optics and haptics. The clear central region, the homogeneous light scattering region (between arrows) and the outer pearl-like regions can be seen. Frame area $4 \times 3 \mathrm{~mm}$. (b) Phase contrast image of the central region of the optics. The sharp interface (arrows) between the clear zone and the homogeneous ghost zone is more clearly seen at higher magnification. The regular cobblestone appearance of the structures in the homogeneous zone is shown in the inset. Frame size $1.0 \times 0.75 \mathrm{~mm}$, inset bar $=10 \mu \mathrm{m}$. (c) Phase image of globular and pearl-like deposits between the optics in the outer zone. Frame size as (b). (d) Immunofluorescence, pseudocolour confocal image of an area of pearl-like tissue adhering to the outer region of a separated optic. Arrows delineate the optic edge. F-actin is encoded red and a-sma is green. Note that there is little overlap (yellow) of the actin isoforms and that the a-sma is distributed preferentially at the outer margins. Bar $=200 \mu \mathrm{m}$. (e) DAPI staining of nuclei remaining in adherent tissue on a separated IOL. The optic appears dark blue, the tissue lighter blue, and the nuclei as bright blue dots (arrow). Tissue adhering to the haptics (arrowheads) was intensely stained and showed the presence of sheets of cells. Frame size $3.5 \times 2.3 \mathrm{~mm}$. (f) Some tissue areas (like the one near the optic edge arrowed in (e) contained degenerate nuclei. Frame size $145 \times 100 \mu \mathrm{m}$.

were variable in shape and size (not shown), had irregular nuclei, and had formed multilayers (Fig 4d). All the cells in this region were positive for $\alpha$-sma expression (Fig 4c). In contrast, on the capsule between the ridge and the equatorial region the epithelial-like cells formed a regular monolayer with round or ovoid nuclei (Fig 4e) and hexagonal mid-cell borders of F-actin (Fig 4f, boxed area). These features are characteristic of normal human LECs. ${ }^{9}$ Only a small number of scattered cells in this monolayer showed $\alpha$-sma expression (Fig 4c and f). Cells clinging to the haptics, which had been pulled out from the inner regions of the bags were positive for vimentin (not shown), indicating their true lens epithelial nature. A schematic diagram summarises the anatomy and cell biology (Fig 5).

\section{Discussion}

Interlenticular (or interpseudophakos) opacification can develop in some eyes with "in the bag" piggyback IOLs. The true incidence is not known, as it tends to occur more than 1 year after surgery. Gayton and Apple have reported an incidence of $43 \%$ in 30 eyes with acrylic IOLs and $22 \%$ in 31 eyes with PMMA lenses followed for at least 2 years. ${ }^{10}$

Roy observed ILO in six out of 27 eyes with AcrySof IOLs ${ }^{11}$ and Shugar and Schwartz in one of 14 eyes with plate haptic silicone IOLs. ${ }^{6}$ ILO is associated with hyperopic shift, the 
mechanism of which is not clearly elucidated. The amount of the refractive shift is higher in acrylic than in PMMA IOLs and it has not been reported yet in silicone IOLs. ${ }^{6}$ Several reasons have been suggested to explain this phenomenon. ${ }^{461213}$

Removal of interlenticular opacities is a challenging process. Elschnig pearl-type material can be easily removed from the interface. However, membrane-type opacities, which can be
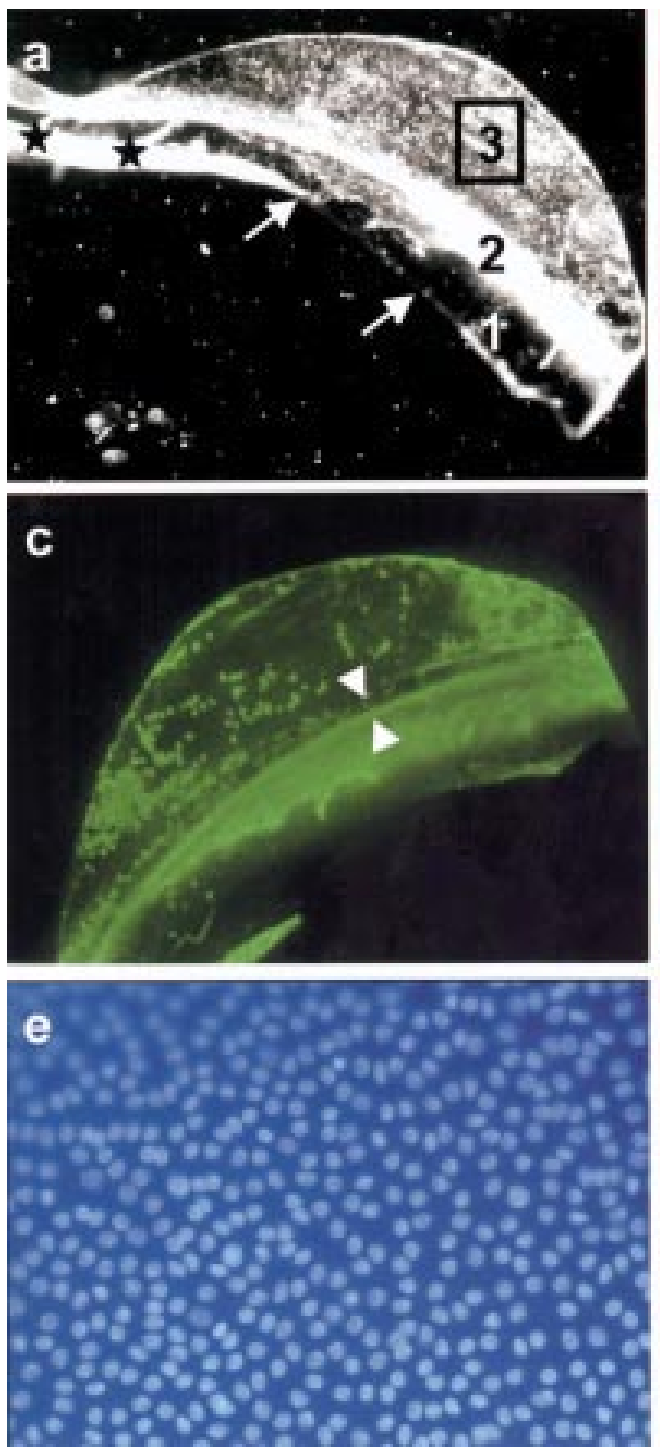

stripped surgically from the PMMA IOLs ${ }^{4}$ without difficulty, are very adherent to the AcrySof IOLs necessitating lens exchange. ${ }^{5}$ Creation of a large corneal or limbal wound and removal of the IOLs en bloc avoids the more time consuming and traumatic intraocular separation or division of the lenses. Explantation of AcrySof IOLs can be difficult owing to the adherence to the anterior capsule. This tight apposition ties in with the ridge configuration
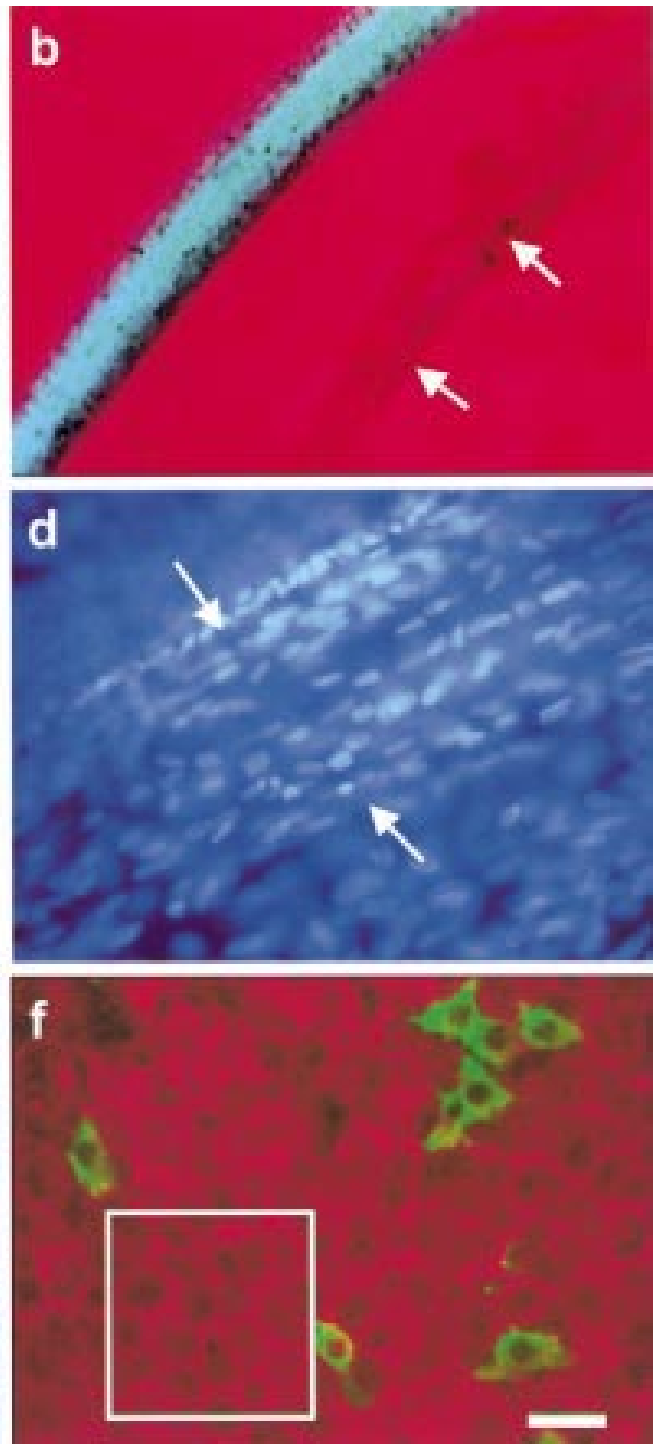

Figure 4 Images of the anterior capsule specimen. (a) Low magnification phase image of the whole fragment before staining. Three regions with quite different characteristics were identified. Region 1 extends from the capsulorrhexis (arrows) to a distinct thickened ridge (2) which forms a bright region along the length of the specimen. This ridge marked the edge of contact with the IOL. Region 3, from the ridge towards the equatorial (non-IOL) region of the capsular bag was evenly covered with cells. (A tissue fragment (starred), which appeared to have been attached to another tissue within the capsular bag, was present at one end of the specimen.) Frame size $4 \times 3 \mathrm{~mm}$. (b) Polarising microscopy pattern obtained from the capsular fragment when viewed through a polariser and analyser set at $90^{\circ}$ and a first order red plate set at $45^{\circ}$ to both axes. The blue birefringence colour results from the ordered alignment of cells (and probably molecules such as collagen) in the region of the prominent ridge (region 2 in (a)). In the red areas there is no significant degree of order in the system. Arrows mark the rhexis edge. Frame $1 \times 0.75 \mathrm{~mm}$. (c) Whole fragment stained for $a$-sma. The distinct ridge (between arrowheads; see also region 2 in (a)) marks the interface between capsule overlying the IOL optic (region 1 in (a)) and capsule facing the interior space between capsular leaflets (region 3 in (a)). Cells in region 1 are all $\alpha$-sma positive, whereas only are small number in region 3 are positive. Frame $3.7 \times 2.5 \mathrm{~mm}$. (d) DAPI staining of nuclei of the ridge region (between arrowheads in (c). The ridge passes diagonally through the centre of the micrograph (between arrows) and contains multilayers of elongated, aligned nuclei. A row of round epithelial nuclei and other out of focus nuclei are on the inner side (top left and region 3 in (a)), and multilayers of elongated/oversize/misshapen nuclei on the outer side (bottom right and region $1 \mathrm{in}(\mathrm{a})$ ). Frame size $145 \times 100 \mu \mathrm{m}$. (e) DAPI staining of the monolayer of nuclei of epithelial cells in region 3. The oval regular nuclei appear identical to those found on a freshly excised normal donor lens epithelium. Frame size as (d). (f) Immunofluorescence, pseudocolour confocal image of cells from the epithelial layer in region 3. F-actin is encoded red and a-sma green. Note the regular hexagonal F-actin outlines (boxed area) of the normal epithelial cells, and these are exactly as they would have appeared in a native, unoperated lens capsule. A few scattered cells (see also Fig 3c) show a-sma expression. Bar $=25 \mu \mathrm{m}$. 


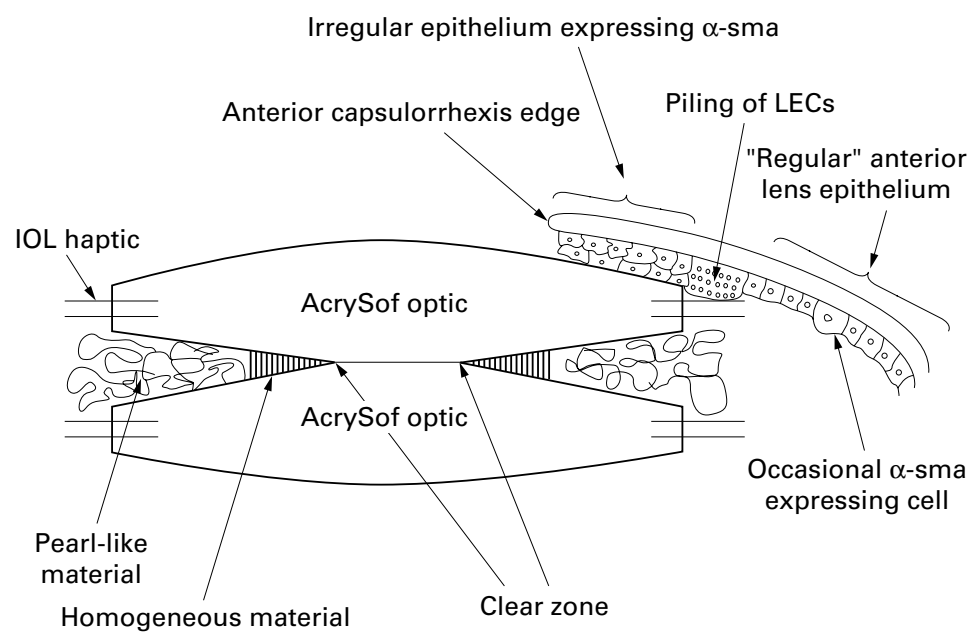

Figure 5 Schematic drawing (AcrySof, not to scale) of the juxtaposition of the piggyback complex and adjacent anterior capsule demonstrating the cell biology of ILO and anterior capsular histopathology. eosin studies, has shown two histological types ${ }^{4}$ and we were able largely to confirm these findings. Two distinct histological types of ILO were observed in these specimens - that is, the Elschnig pearl-type consisting of proliferating/ retained LECs in the interlenticular space and the amorphous membranous-type consisting of ECM deposits with the same cobblestone appearance given by LECs. ${ }^{4}$ To the best of our knowledge no detailed immunological studies of cell types/viability have been reported before.

The pearl-type material in the present specimens was found to consist of swollen and globular live cells with an F-actin cytoskeleton and nucleus. They showed evidence of some form of transformation/differentiation in that some expressed $\alpha$-sma, and some had degenerate nuclei. While these cells would be unlikely to undergo cell division the process of globular swelling could be ongoing, increasing the amount of material. The membranous region of opacification was not amorphous but showed a distinct hexagonal pattern with similar dimensions to LECs. Since no live cells were present this most likely represents ghost cells - that is, devitalised cells, within a sheet of extracellular matrix produced by the previously live lens epithelial cells.

The cellular material in the anterior capsule fragment reveals viable LECs despite the time interval from the operation, similar to those seen in eyes with single IOLs. ${ }^{8}$ The close apposition of the anterior capsule and anterior IOLs periphery leads to distortion of epithelial cell alignment, which in turn results in the build up of multiple layers of cells and ECM at the interface between the IOL edge and the capsule, forming the thickened ridge. Interestingly, the cells residing in the area between the ridge and the capsulorrhexis are irregular and all express $\alpha$-sma, indicating a transformation to a fibroblastic cell type. In contrast, cells in the area between the ridge and the bow are regular and only a few cells express $\alpha$-sma. These cells have the potential to proliferate and colonise any surfaces and spaces within the capsular bag leading to further accumulation of opacified material. Piling of the cells at the ridge could lead to a diversion of the cells into interlenticular space and further proliferation to form Elschnig pearl and globules. These structures are very similar to those seen in capsular opacification with single IOL implantation. ${ }^{9}$

It is very likely that an initial small central contact zone is created at the time of implantation by the compression of the IOLs owing to limited space in the capsular bag. This compression would inhibit encroachment of LECs into this central region. However, LECs are able to proliferate and lay extracellular matrix in the interlenticular space up to the edge of the contact zone. Opacification of the anterior capsule overlapping the optic and fusion of the peripheral anterior and posterior capsule by proliferating cells ${ }^{15}$ result in added anterior-posterior contraction forces, further compression of the IOLs, and hence expansion of the contact zone. In addition to the 
compression forces exerted on the IOLs by the capsular bag, ECM laid by the proliferating cells may act as a biological "glue" attracting the optic surfaces even closer causing more entrapment and death of the epithelial cells within the expanded contact area. Expansion of the central contact zone with the progressive flattening of the opposing surface of the two IOLs would lead to gradual reduction of the refractive power in the central area of the IOL complex and hyperopic shift. As the paracentral and peripheral vision are obliterated by the opacification in these areas (Fig 2) the patient is forced to use the central contact area for vision. Owing to flattening this area has less refractive power resulting in hyperopic shift. ${ }^{12}$ The opacified areas also cause glare.

\section{Conclusions}

The tight apposition of the anterior lens capsule to the anterior optical surface of the anterior piggyback IOL results in the formation of a closed system, bounded by the edge of the IOL/capsular ridge and encompassing within it the remainder of the capsule and the interlenticular spaces and surfaces. This closed environment between the anterior and posterior capsules is much larger than that formed by a single IOL and so there is a greater volume in which material can accumulate. Significantly, there are also more surfaces upon which material can be deposited. Cellular proliferation, deposition of ECM from proliferating LECs and capsular changes induced by cell metaplasia may lead to ILO formation and hyperopic shift in AcrySof IOLs. Careful separation of the AcrySof IOL complex from the capsule, meticulous clean up of the proliferating material, and implantation of a single or dual in the bag PMMA IOLs through a large incision with capsulorrhexis enlargement may help in the prevention of recurrence of interface opacification.
A free paper entitled "Interlenticular opacification in piggyback AcrySof intraocular lenses: a histopathological study" and a video entitled "Interlenticular opacification in piggyback Acrysof intraocular lenses: surgical technique for exchange of IOLs" containing parts of the submitted manuscript were presented at the UKISCRS 2000 annual meeting in Chester,
UK.

The authors wish to acknowledge the referring consultant ophthalmic surgeon Mr David Garlick.

1 Gayton JL, Sanders VN. Implanting two posterior chamber intraocular lenses in a case of microphthalmos. 7 Cataract Refract Surg 1993;19:776-7.

2 Holladay JT, Gills JP, Leidlein J, et al. Achieving emmetropia in extremely short eyes with two piggyback posterior chamber intraocular lenses. Ophthalmology 1996;103:1118-23

3 Shugar JK, Lewis C, Lee A. Implantation of multiple foldable acrylic posterior chamber lenses in the capsular bag for high hyperopia. F Cataract Refract Surg 1996;22: 1368-72

4 Gayton JL, Apple DJ, Peng Q, et al. Interlenticular opacification: clinicopathological correlation of a complication of posterior chamber piggyback intraocular lenses. $\mathcal{F}$ Cataract Refract Surg 2000; 26:330-6.

5 Shugar JK, Keeler S. Interpseudophakos intraocular lens surface opacification as a late complication of piggyback acrylic posterior chamber lens implantation. $\mathcal{F}$ Cataract Refract Surg 2000; 26:448-55.

6 Shugar JK, Schwartz T. Interpseudophakos Elschnig pearls associated with late hyperopic shift: a complication of piggyback posterior chamber intraocular lens implantation. $f$ Cataract Refract Surg 1999;25:863-7.

7 Shugar JK. Interpseudophakos opacification. Annual Meeting of the American Academy of Ophthalmology, Orlando, ing of the American Academy

8 Marcantonio JM, Vrensen GFJM. Cell biology of posterior capsular opacification. Eye 1999; 13:484-8.

9 Marcantonio JM, Rakic JM, Vrensen GFJM, et al. Lens cell populations studied in human donor capsular bags with $2000 ; 41: 1130-41$.

10 Gayton JL, Apple DJ. Refractive stability and long-term interlenticular membrane formation of piggyback intraocu-
lar implants. Annual Meeting of the American Academy of Ophthalmology, Orlando, Florida, USA, October 1999.

11 Roy F. Membrane formation in primary piggyback intraocular lenses. American Society of Cataract and Refractive Surgery, Boston, Massachusetts, USA, May 2000.

12 Findl O, Menapace R. Piggyback intraocular lenses. 7 Cataract Refract Surg 2000;26:308-9.

13 Findl O, Menapace R. Piggyback intraocular lenses. 7 Cataract Refract Surg 2000;26:308-9; discussion by Shugar JK, 309.

14 Hull CC, Liu CSC, Sciscio A. Image quality in polypseudophakia for extremely short eyes. Br F Ophthalmol 1999;83: $656-63$

15 Liu CSC, Wormstone IM, Duncan G, et al. A study of human lens cell growth in vitro. A model for posterior capsule opacification. Invest Ophthalmol Vis Sci 1996;37:90614. 